\title{
The analysis of the canid mitochondrial genome studied in Morocco shows that it is neither wolf (Canis lupus) nor Eurasian jackal (Canis aureus)
}

The mitochondrial genome of three Moroccan canids has been analysed. Two of them complete and one partial. The sequences are included in GenBank with the accession number KT378605 (16721 bp), KT378606 (16734bp) and KT378607 (27809bp) Theses results have been compared with the results currently available in GenBank. In the phylogenetic analysis of the of cytochrome $b$ regions and control region the three are grouped together with Canis lupus lupaster and the Senegalese golden jackal Canis aureus and separate from the wolf Canis lupus and the Eurasian golden jackal Canis aureus. The comparison of the complete mitochondrial genomes with Canis lupus confirms the distance between the two groups. We conclude that they belong to a different species to the wolf Canis lupus and the Eurasian golden jackal. We propose in agreement with (Koepfli et al., 2015) that it's scientific name be Canis anthus by merit of being the name by which it was classified and published for the first time as a different species by Cuvier in 1824 .

The preprint is a translation of a Spanish document published in Altorero journal on the 15th August 2015. 


\title{
The analysis of the canid mitochondrial genome studied in Morocco shows that it is neither wolf (Canis lupus) nor Eurasian jackal (Canis aureus).
}

\author{
Vicente Urios ${ }^{1}$, María P. Donat-Torres ${ }^{2}$, Carlos A. Ramírez Castillo ${ }^{1}$ Octavio Monroy-Vilchis $^{3}$, Hamid Rguibi \\ Idrissi $^{4}$
}

\author{
1 .Investigation Group of Vertebrate Zoology. University of Alicante, Alicante, Spain. \\ 2 .Research Institute for Integrated Coastal Zone Management. Polytechnic University of Valencia, Valencia, Spain \\ 3. Biological Research Center of Applied Science. National Autonomous University of Mexico, Mexico DF. Mexico \\ 4. Faculty of Science, Chouaib Doukkali University , El Jadida, Morocco
}

Corresponding author: vicenteurios@yahoo.es, mpdonat@eaf.upv.es, carlostamandua@gmail.com

\begin{abstract}
The mitochondrial genome of three Moroccan canids has been analysed. Two of them complete and one partial. The sequences are included in GenBank with the accession numbers KT378605 (16721 bp), KT378606 (16734bp) and KT378607 (27809bp) Theses results have been compared with the results currently available in GenBank. In the phylogenetic analysis of the of cytochrome b regions and control region the three are grouped together with Canis lupus lupaster and the Senegalese golden jackal Canis aureus and separate from the wolf Canis lupus and the Eurasian golden jackal Canis aureus. The comparison of the complete mitochondrial genomes with Canis lupus confirms the distance between the two groups. We conclude that they belong to a different species to the wolf Canis lupus and the Eurasian golden jackal. We propose in agreement with (Koepfli et al., 2015) that it's scientific name be Canis anthus by merit of being the name by which it was classified and published for the first time as a different species by Cuvier in 1824 .
\end{abstract}

Key Words: Canis anthus, Canis aureus lupaster, Canis lupus lupaster, Canis lupaster, complete mitochondrial genome, North African jackal, Morocco. 


\section{INTRODUCTION}

The presence of African canids of difficult taxonomic assignation has been identified for a long time. In recent years the three publications that proposed the North African jackal, Canis aureus lupaster, was not a jackal but a wolf have focused their interest on the taxonomic status of these canids. The genetic analysis of mitochondrial sequences by (Rueness et al., 2011) and (Gaubert et al., 2012) together with the appearance of the photography published by (Gaubert et al., 2012) and (Urios et al., 2012) would suggest that it was not a jackal (Canis aureus) and its membership of a subspecies of lineage Canis lupus.

The confirmation thanks to these initial genetic studies, observations and photography, of the existence on the African continent of a taxon different to the Eurasian jackals and wolves is of great interest and was a first step towards a better understanding of these canids.

The biogeographic isolation of the African canid from the Eurasian species and its different morphology made us consider the hypothesis that the process of separation of this lineage went beyond the level of subspecies. To elucidate this question a wide ranging study has been carried out in Morocco in which by means of photo traps more than fifty examples were recorded and the mitochondrial genome of three examples was analysed, two of them complete. They were compared with the available GenBank sequences of Canis lupus and Canis aureus from Eurasia.

\section{MATERIAL AND METHODS}

The Haut Commissariat aux Eaux et Forêts et à la Lutte Contre la Désertification of Morocco Kingdom supported our study by means of several investigation permits (Decision No 219/2011, 267/2012, 18/2013, 36/2015 HCEFLCD/DLCDPN/DPRN/CFF).

Samples have been collected since 2012, proceeding from examples found knocked down dead. The results obtained from three analysed individuals are shown. Two of the individuals 
(Can 3 and Can 6 collected in 2012) come from the Atlas Mountains and the third (Can 13, collected in 2013) come from the Sahara Desert.

For DNA extraction epithelial tissue from the footpad area was used (Can 3), muscular tissue (Can 6) and hair (Can 13). The extractions as well as the PCR reactions were done separately for each individual.

Two methods were used for the extractions: extraction by use of a kit with proteinase and extraction by means of Chelex and proteinase K. In the first case the manufacturer's (Invisorb) protocol was followed apart from leaving the sample all night with the lisis buffer and proteinase $\mathrm{K}$ at an initial temperature of $56^{\circ} \mathrm{C}$, closing the bath afterwards. The next day it was incubated at $56^{\circ} \mathrm{C}$ for 5 hours, after which protocol was followed.

When Chelex 100 sodium was used previously a restriction digest was done with proteinase K (Gagneux, Boesch, and Woodruff 1997); $20 \mu \mathrm{l}$ of proteinase $\mathrm{K}$ were added (of concentration $10 \mu \mathrm{g} / \mu \mathrm{l}$ ) to $100 \mu \mathrm{l}$ of water containing $2 \mathrm{~cm}$ of hair with follicle (cut into very small fragments) previously treated with PBS (phosphate buffered saline); leaving the sample all night with the lisis buffer and proteinase $\mathrm{K}$ at an initial temperature of $56^{\circ} \mathrm{C}$, then turning off the bath. The next day at a temperature of $56^{\circ} 100 \mu \mathrm{l}$ of Chelex sodium 100 was added at $10 \%$ and was incubated for two hours. Vortex 10s was applied, it was placed in boiling water for 10 minutes, vortex 10s was applied once again, it was centrifuged for 3 minutes at maximum spin and the supernatant was extracted (Palomares et al., 2002)

Basically three mixes of products were used for the PCR reactions depending principally on the polymerases that were used (5Prime, Mobiolab and Pangea made by Canvax). Manufacturer's instructions were followed for the quantities of each product used in the reaction.

In the three canids mitochondrial DNA regions were analysed about which more information exists in GenBank so that the results obtained could be compared: Cytochrome b (Cyt b) and (D-loop) control region. Also the complete mitochondrial genome was obtained from the two most geographically close canids in order to compare their mutual resemblance.

For amplification two groups of primers were used. As the first group, for the amplification of the complete mitochondrial genome the primers indicated by (Björnerfeldt, Webster, and Vilà 2006) were used, with which 37 fragments were obtained. The primers F35a and R35 
did not work and were substituted for the primers F35b and R35b (Björnerfeldt, Webster, and Vilà 2006)

A second group of primers habitual to the bibliography was also used for particular fragments: for 12S rRNA the primers L01091 and H01478 (Kocher et al., 1989); for 16S rRNA the primers W16S_F and W16S_R; for Cyt b the primers cytb-1 (Gaubert et al., 2012) cytb-2 (Janczewski et al., 1995); and for the D-loop region the primers CR1F and CR2R (Palomares et al., 2002). The fragments amplified with this second group of primers turned out to be shorter in length than their corresponding equivalents from the first group of primers.

Electrophoresis was done with agarosa gel at 1.5\% using TBE $5 \mathrm{X}$ as a buffer at $1.5 \%$; RedSafe was used as a developer and there was always a well with Generuler 100 bp plus. The amplification was repeated up to 5 times when necessary.

The sequencing was done by Macrogen. The sequencing reactions were done in the Peltier Thermal Cycler DNA Engine tetrad 2 (BIO-RAD) using the ABI Big Dye ${ }^{\circledR}$ Terminatorcycle sequencing kit v3.1 (Applied Biosystems), following the protocols supplied by the manufacturer and using the corresponding primer.

The list of accession numbers used to compare the material in the present study they were obtained from the GenBank database (http://www.ncbi.nlm.nih.gov) (Benson et al., 2006). The nomenclature with which appear the taxons in this document is that which is listed in the accessions respective to GenBank.

Initial information for the phylogenetic study was acquired from the sequences obtained and their possible similarity with those available in GenBank, using (BLAST 2015) (Altschul et $a l ., 1990)$. The assembly of the sequences was done with the program Sequencer 4.1.4. (Gene Codes Corporation, sequence analysis software, Gene Codes Corporation, Ann Arbor, Michigan, USA) and with Bioedit 7.5.2 (http://bioedit.software.informer.com/) (Hall 1999). The alignments of the nucleotide sequences were done with Bioedit 7.2.5 and with the version of Clustal (Thompson, Higgins, and Gibson 1994) available in said program. It was completed manually. The MEGA6 program (Tamura et al., 2013) was used to calculate the genetic distances utilizing the Kimura 2-parameter model (Kimura, 1980) and the Tamura Nei model (Tamura and Nei, 1993), as well as to analyse models and phylograms. The program DnaSP 5.10 (Librado and Rozas 2009) was used to calculate the number of polymorphic sites (S), nucleotide diversity $(\pi)$, the number of haplotypes (h) and haplotype 
diversity (Hd). The parameter $\theta$ was calculated with MEGA6. The most suitable evolutionary models for the complete evolutionary genomes were calculated using jModel Test (Darriba $e t$ al., 2012). The phylograms were done with the programs BEAST1.7 (Drummond et al., 2012) (Rambaut et al., 2014). The time elapsed to the most recent common ancestor (TMRCA) was calculated with the program BEAST 1.7 using Bayesian Markov Chain Monte Carlo (MCMC). The divergence time between wolf and coyote which was used as external group was calculated in 1 MYA (Wayne et al., 1997) (Vilà et al., 1999) (LindbladToh et al., 2005).

\section{RESULTS}

The sequences were obtained in 2013 and 2014; they are included in GenBank and the accessions are: Can 3: accession KT378605, of 16721bp; Can 6: accession KT378606, of 16734bp; Can13: accession KT378607, of 2789bp. As can be seen from their size, the first two are complete mitochondrial genomes and the last one partial.

Firstly, before doing the first phylogenetic analysis our three individuals were compared to each other. For this the shared fragment was used that is comprised of 2776 bases corresponding to the continuous partial zone of mitochondrial DNA that consists of: gene ND5,ND6, tRNA-Glu, Cyt b, tRNA-Pro and D-loop.

Table 1 shows the matrix of distances found between them. The number of bases per site are below the diagonal and the deviation standard above it. The individuals Can 6 and Can 13 present the smallest distance between them all despite that the closest geographically are the two from the Atlas Mountains Can 3 and Can 6, while Can 3 and Can 13 are the most distant genetically. With respect to polymorphic sites (S) their number is 72, nucleotide diversity $(\pi)$ is 0.01730 and the value of Theta $(\theta)$ is 0.001095 . The data clearly shows membership of the same taxon even though the fragment compared includes the D-loop region variable. 
NOTPEER-REVIEWED

\begin{tabular}{|l|c|c|c|}
\hline & Can 3 & Can 6 & Can 13 \\
\hline Can 3 & & 0,0029916502 & 0,0043414981 \\
\hline Can 6 & 0,0172230232 & & 0,0030475545 \\
\hline Can 13 & 0,0286723005 & 0,0161844018 & \\
\hline
\end{tabular}

Table 1. Genetic distances between the three canids studied. Model TN93. The standard deviations are shown above the diagonal.

When this study was done the availability of the data in GenBank about the canids that could be phylogenetically closest to ours was scarce (excepting Canis lupus for which there is ample documentation); there are few mitochondrial gene sequences and also they are partial fragments. It is for this reason that apart from comparing our complete mitochondrial genomes before we have also made several comparisons between only parts of our sequences with the other sequences available in said database. Shorter than ours, they deal with the cytochrome b and D-loop regions.

\section{Analysis of Cytochrome b regions (Cytb) and the (D-loop) Control Region of the Canis lupus lupaster / aureus african group.}

Next are detailed the results of comparing sequences Cytb + control region (680bp) of our three individuals with accession that can be found in GenBank as $C$. lupus lupaster, used by Rueness et al., 2011 and Gaubert et al., 2012; and accession of C. aureus from Senegal (Gaubert et al., 2012) since the most recent accession of (Vasco, 2012) are not listed, Rueness et al., 2015 and Koepfli et al., 2015. The set of 13 examples has a polymorphic site number (S) of 33, the nucleotide diversity number $(\pi)$ is 0.012898 and the value of Theta $(\theta)$ 0.016064 .

The genetic distances are very small varying between 0 and 0.029 . The smallest distance is given between our Can 6 and one from Algeria, being 0; the two Senegalese also have a distance of 0 between them. The greatest distance is given between the Canis aureus from Senegal and the individual from Egypt (Rueness et al., 2011) this individual being in general more distant from the rest. The genetic distance between all of them is low, at 0.013. All in all the values manifest that the canids analysed by us fit in the group well. 


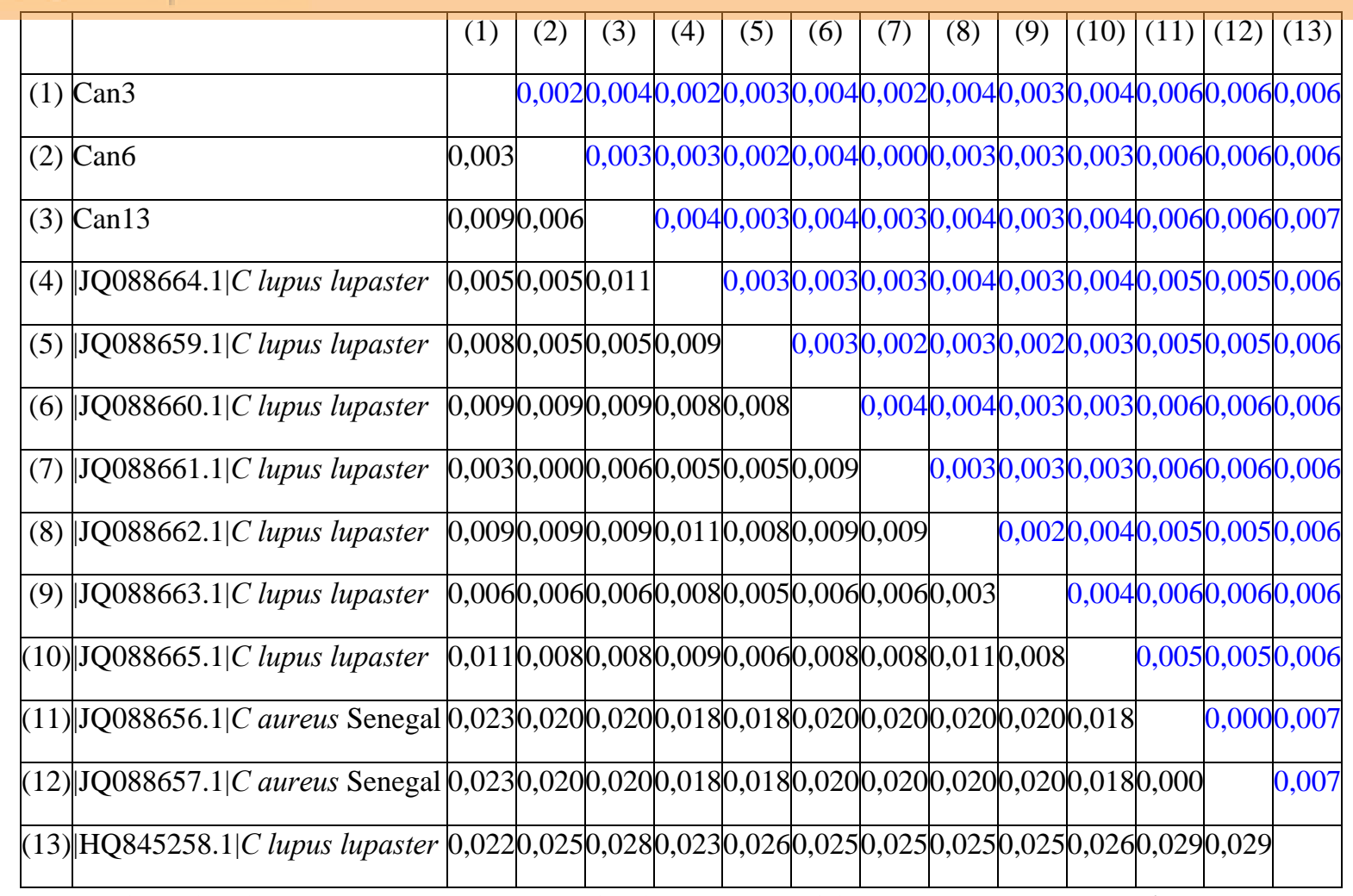

Table 2. Genetic Distance between the Three Canids and the African group Canis Lupus lupaster / aureus Kimura 2 model. The standard deviations are shown above the diagonal.

\section{Analysis of Cytochrome b Zones (Cytb) and the (D-loop) Control Region of the Canis lupus lupaster / aureus african Group Compared with the Genus Canis}

To compare the distance between the different taxons of the clado lupus fragments of cytochrome b (Bradley and Bake 2001) from the control region (610 pb) have also been used. The accession numbers are enumerated in Table S3 of the appendix. The phylogenetic tree was inferred using the Maximum Verisimilitude method and the Kimura 2 Model in the MEGA 6 program. The African lupus lupaster / aureus group together with those of this study remain distinguished from the rest of the wolves on the one hand and from Eurasian Canis aureus on the other, that seems more primitive. Although the African canid is more related to Canis lupus than to Canis aureus. However it must be pointed out that in phylograms made only with cytochrome b we have obtained a greater age for Eurasian Canis aureus than for Canis latrans. 


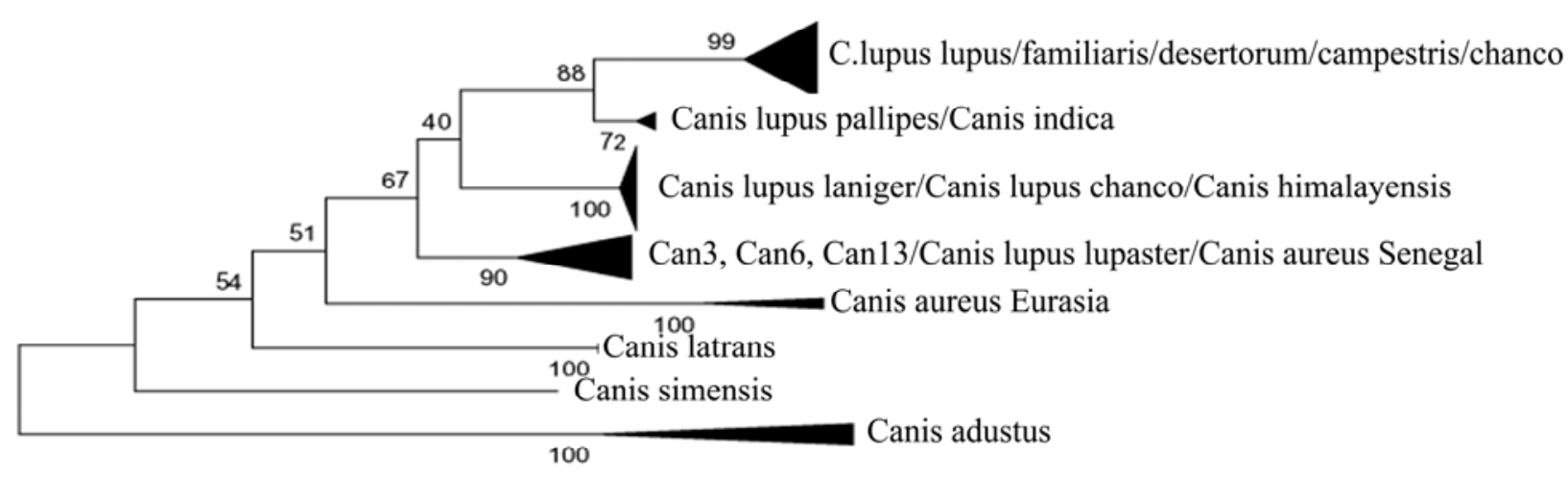

Figure 1. Phylogenetic Tree obtained with the Maximum Verisimilitude Method. Kimura 2 model. The numbers indicate the bootstrap value.

\section{Analysis of the Complete Mitochondrial Genomes}

Shown next are the results of comparing the data of the complete mitochondrial genomes of Can 3 and of Can 6 with the data of the Canis lupus lineages available in GenBank, using Canis latrans as an external group. Canis lupus lupus and Canis lupus familiaris were annoted with the common inscription Canis lupus lupus / familiaris. In table S1 of the appendix appear the genetic distances between them as measured by the differences in base pairs. The accessions are noted in table 2 of the appendix. The distance between the individuals of group Can 6 and Can 3 and the rest of the wolves varied between 0.042 and 0.05; being as a group the furthest from the rest of the groups of wolves.

This is reflected with great clarity in the phylogenetic trees. In the phylogenetic tree obtained with the Maximum Verisimilitude Method (Figure 2) the Can 3 and Can 6 group is a separate lineage from Canis lupus and more primitive. Inside Canis lupus group C. lupus laniger and C. lupus chanco are split from the rest, except for one individual from C. lupus chanco that shows different behaviour and is grouped with the rest of $C$. lupus. 


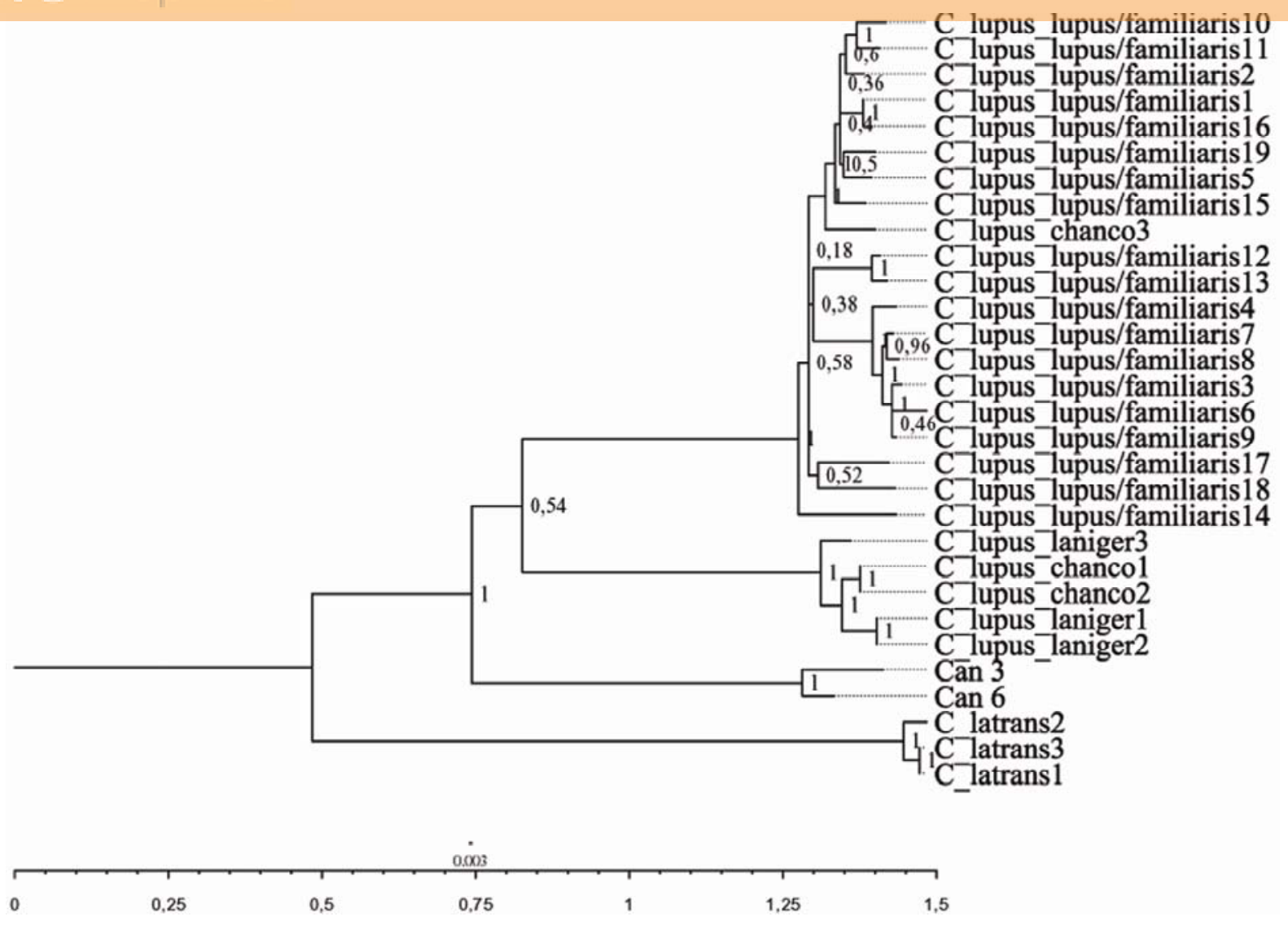

Figure 2. Phylogenetic Tree obtained with the Maximum Verisimilitude Method and the TrN $+\mathrm{I}+\mathrm{G}$ model. The nodes show the bootstrap value and the timescale (MYA)

Firstly the jModeltest was used to discover which is the model most suitable to create the phylogenetic tree by Bayesian inference. Resulting in the conclusion that the model is $\operatorname{TrN}+\mathrm{I}+\mathrm{G}$ for both the BIC criteria as well as the AIC criteria. The result achieved using BEAST for said model is shown in Figure 3 with p-inv 0.7320 and gamma shape1.0350. The same as with the prior phylogram a greater age is observed for Can 3 and Can 6 than for $C$. lupus. The lineage of laniger and chanco is completely grouped together, which could give an idea about the reliability of the adjustment of the tree. 


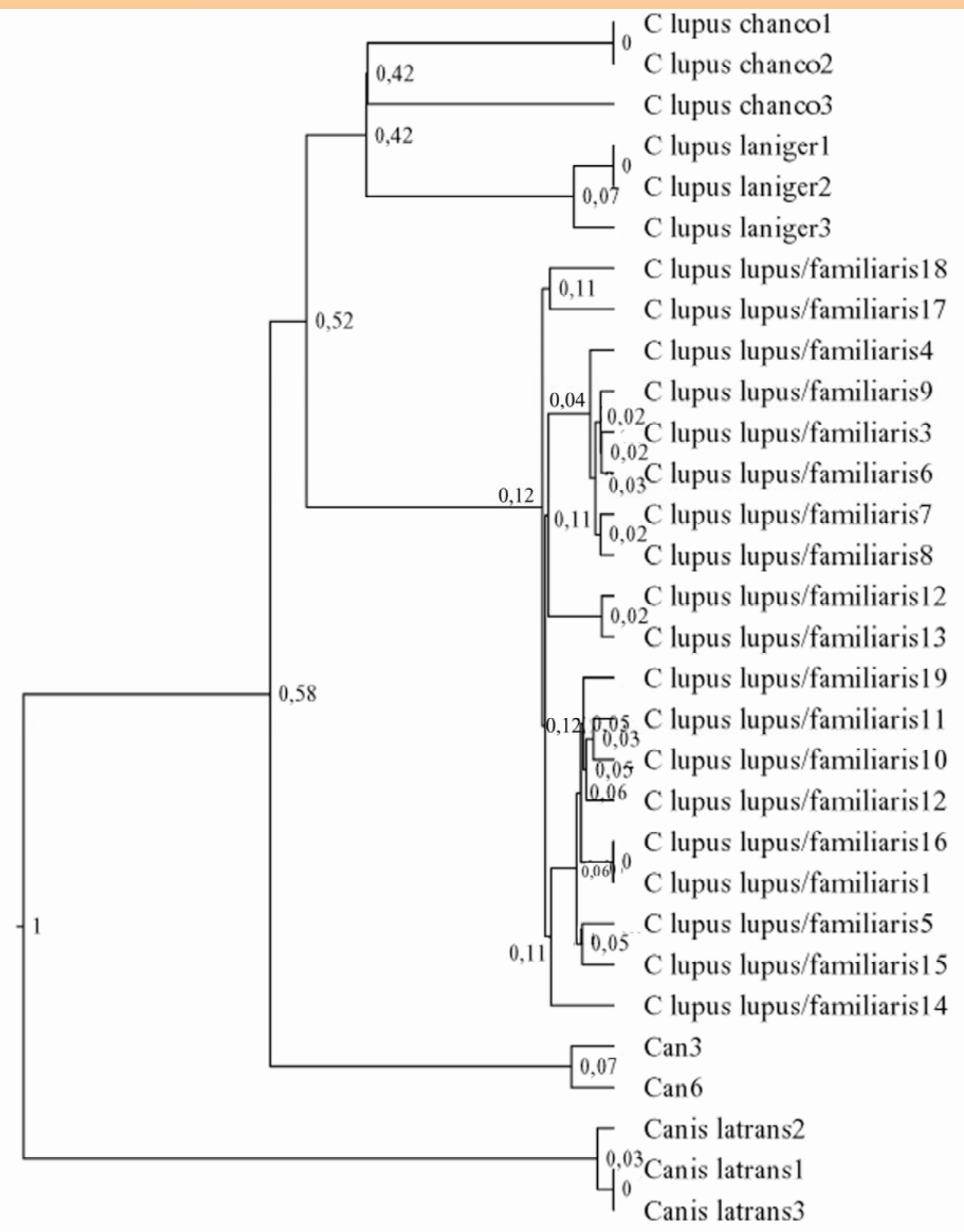

$\longrightarrow 0,25 \quad 0,5+1$

Figure 3. Phylogenic Tree obtained by Bayesian analysis with the BEAST program and the $\operatorname{TrN}+\mathrm{I}+\mathrm{G}$ model. The numbers of the nodes and the scale show the time (MYA). 


\section{DISCUSSION}

The data from these genomes that we provide in this work manifest that these Moroccan canids surely deserve to be categorised as a different species to the golden jackal Canis aureus and the wolf Canis lupus. As we will see next this has already been stated since the $19^{\text {th }}$ century based on morphological studies. In reference to more current molecular studies published on the internet, the base sequences do not appear in GenBank for which reason we have not been able to include them in this study for the purpose of comparison. Ruenes et al., 2015 says that it is not a hybrid and is distinct from the golden jackal $C$. aureus and the wolf C. lupus, being a unique taxon, naming it as Canis aureus lupaster, without assigning it a specific category. Koepfli et al., 2015 in an exhaustive study in which examples from Morocco are also included comes to the same conclusion as us that it is a species different from Canis lupus and Canis aureus.

The perception that the so called African jackals and African wolves were something genuine to that continent and different to the Eurasian has been taken up by various scientists and naturalists for three centuries, considering them either jackals, Canis aureus, or wolves Canis lupus, or other different species.

Currently genetic studies have provided a new tool to corroborate what different scholars have considered and how different conclusions are expressed in the utilization of varied nomenclature as will be seen next.

\section{Consideration as a Golden Jackal Canis aureus lupaster}

Since it was first described it has been considered a subspecies of the golden jackal, Canis aureus; it is already cited in this way, Canis aureus lupaster (Hemprich \& Ehrenberg 1833) when these authors initially described it as Canis lupaster.

Cabrera in 1932 following (Oken 1815-1816) uses the generic name of Thos to refer to jackals, describing various subspecies between them Thos lupaster marocannus that would be synonymous with Canis aureus marocannus. Schwarz in 1926 refers to the great canid of North Africa and Sinai as Canis aureus lupaster, the same as Ellerman and Morrison-Scott in 1951 they give as good the name Canis aureus lupaster (Hemprich\& Ehrenberg 1933) and as 
synonyms Canis Lupaster (Hemprich and Ehrenberg 1933) and Canis Sacer (Hemprich and Ehrenberg 1933). Corbet in 1978 and Osborn and Helmy in 1980 they also named it as Canis aureus lupaster. In 1982 it still appears on the world checklist of mammals as a subspecies of golden jackal (Canis aureus) (Hocnaki, Inman and Koeppl 1982) And Wozencraft 2005 also speaks of $C$. aureus lupaster.

\section{Consideration as an African wolf Canis lupus lupaster}

The first biologist to classify the canid lupaster as a subspecies of C. lupus is (Huxley 1880) on noting the similarities between the crania of lupaster and Indian wolves. It is not until 1981 that the zoologist Walter Ferguson of University of Tel Aviv considered lupaster as a subspecies of $C$. lupus on the basis of cranial measurements saying that classification of this animal as a jackal owes exclusively to its small size (Ferguson 1981). He says that in the Sinai to the North East of Egypt C. aureus lupaster must be considered as C. lupus lupaster.

Subsequently Rueness et al., 2011 says that the Egyptian and Ethiopian C. aureus lupaster must be considered a subspecies of wolf on the basis of genetic distance. His results placed it as more distant from the Eurasian golden jackal Canis aureus.

Gaubert et al., 2012 on the basis of genetic analysis of cytochrome b and the control region also classify it as such in Mali, Senegal, Algeria and Tunisia.

\section{Consideration as a Different Species}

In a continent with the presence of currently existing very primitive canids and others extinct (Geraads, 2011) the first time that the species C. lupaster appears is in 1832 classified as a different species by Hemprich and Ehrenberg being the type locality El Fayum (Egypt). Subsequently in 1902 Anderson recognised the Egyptian species C. Lupaster as a valid species and pointed out the presence of the species in Algeria and Tunisia (Anderson 1902). He identified C. sacer as lupaster, rejected the presence of C. aureus in North Africa and affirmed that C. lupaster is the only wild species that inhabits the region. Hilzheimer in 1908 recognises three species: $C$. sacer, $C$. doederleini in Egypt and C. lupaster.

Flower in 1932 also refers to two different species of jackal in Egypt and to the first he refers to as $C$. lupaster. Kurten in 1965 accredits the presence of $C$. lupaster to places in North 
Africa and Palestine in the late Pleistocene. In 1989 Nicolai Spassov declared that the form lupaster has morphological characteristics that maintain lupaster as a different species.

In 1991 Kowalski and Rzebik-Kowalska supported the work of (Heim de Balsac 1936) in which he names $C$. lupaster to the North of Algeria together with other species, $C$. riparius and $C$. variegatus more to the South.

The name Canis anthus was assigned by F. Cuvier to classify the jackal or Senegalese wolf in 1820 which he described at length in his Histoire Naturelle des mammifères (Geoffroy and Cuvier 1824) which has been resumed by Koepfli et al., 2015

Although phylogentetically our results show that it is closer to $C$. lupus than to $C$. aureus, taking into account the criteria of the age we propose the name of Canis anthus, in agreement with Koepfli et al., 2015 Coined by Cuvier before the later Canis lupaster coined by Hemprich \& Ehrenberg. We remain in the hope that the diverse advances being made continue, that a future greater genetic knowledge of African canids could establish the existence of more taxons and that for the most lupoid in form the name lupaster is used.

\section{Acknowledgements}

Our thanks to Maela León of the Mediterranean Agroforestal institute (Politechnic University of Valencia) for her orientations in the laboratory and her data analysis.

\section{REFERENCES}

Koepfli, K.-P., Pollinger, J., Godinho, R., Robinson, J., Lea, A., Hendricks, S., Schweizer, R. M., Thalmann, O., Silva, P., Fan, Z., Yurchenko, A. A., Dobrynin, P., Makunin, A., Cahill, J. A., Shapiro, B., Álvares, F., Brito, J. C., Geffen, E., Leonard, J. A., Helgen, K. M., Johnson, W. E., O’Brien, S. J., Van Valkenburgh, B., Wayne, R. K. (2015). Genome-wide Evidence Reveals that African and Eurasian Golden Jackals Are Distinct Species. Current Biology 25. doi:10.1016/j.cub.2015.06.060.

Rueness, E. K., Asmyhr, M.G., Sillero-Zubiri, C., Macdonald, D. W., Bekele, A., (2011). The Cryptic African Wolf: Canis aureus lupaster Is Not a Golden Jackal and Is Not Endemic to Egypt. PLoS ONE 6(1): e16385. doi:10.1371/journal.pone.0016385. 2011. 
Gaubert, P., Bloch, C., Benyacoub, S., Abdelhamid, A., Pagani, P., et al. (2012) Reviving the African Wolf Canis lupus lupaster in North and West Africa: A Mitochondrial Lineage Ranging More than 6,000 km Wide. PLoS ONE 7(8): e42740. doi: 10.1371/journal.pone.00427. 2012.

Urios, V., Ramírez, C., Gallardo, M. and Rguibi Idrissi, H. (2012). Detectan al lobo en Marruecos gracias al uso del foto-trampeo. Quercus (319): 14-15). 2012.

Gagneux, P., Boesch, C., Woodruff, DS. (1997) Microsatellite scoring errors associated with noninvasive genotyping based on nuclear DNA amplified from shed hair. Molecular Ecology, 6, 861-868.

Palomares, F., Godoy, J.A., Piriz, A., O’Brien, S.J., Johnson, W.E. (2002). Faecal genetic analysis to determine the presence and distribution of elusive carnivores: design and feasibility for the Iberian lynx. Molecular Ecology 11: 2171-2182.

Björnerfeldt, S., Webster, M.T., Vilà, C. (2006). Relaxation of selective constraint on dog mitochondrial DNA following domestication. Genome Res. 16:990-994.

Kocher, T.D., Thomas, W.K., Meyer, A., Edwards, S.V., Paabo, S., et al. (1989). Dynamics of mitochondrial DNA evolution in animals: amplification and sequencing with conserved primers. Proc Natl Acad Sci U S A 86: 6196-6200.

Janczewski, D.N., Modi, W.S., Stephens, J.C., O’Brien, S.J. (1995). Molecular evolution of mitochondrial 12S RNA and cytochrome b sequences in the pantherine lineage of Felidae. Mol Biol Evol 12: 690-707.

National Center for Biotechnology Information (NCBI). 2015. The GenBank database. http://www.ncbi.nlm.nih.gov/genbank/

Benson, D. A., Karsch-Mizrachi, I., Lipman, D. J., Ostell J., and Wheeler, D. L. (2006). GenBank. Nucleic Acids Res. 34:D16-D20.

BLAST. (2015). (http://www.ncbi.nlm.nih.gov/BLAST/).

Altschul, S. F., W. Gish, W. Miller, E. W. Myers, and D. J. Lipman. (1990). Basic local alignment search tool. J. Mol. Biol. 215:403-410. 
Hall, T.A. (1999). Bioedit: a user-friendly biological sequence alignment editor and analysis program for Windows 95/98/NT. Nucleic Acids Symp Series 41:95-98. Available at: http://www.mbio.ncsu.edu/Bioedit/bioed. http://www.mbio.ncsu.edu/Bioedit/bioedit.html.

Thompson, J. D., D. G. Higgins, and T. J. Gibson. (1994). CLUSTAL W: improving the sensitivity of progressive multiple sequence alignment through sequence weighting, positionspecific gap penalties and weight matrix choice. Nucleic Acids Res. 22:4673-4680. .

Tamura, K., Stecher, G., Peterson, D., Filipski, A., and Kumar, S. (2013). MEGA6: Molecular Evolutionary Genetics Analysis Version 6.0. Molecular Biology and Evolution 30: 2725-2729.

Kimura, M. (1980). A simple method for estimating evolutionary rate of base substitutions through comparative studies of nucleotide sequences. Journal of Molecular Evolution 16:111120.

Tamura, K., Nei, M. (1993). Estimation of the number of nucleotide substitutions in the control region of mitochondrial DNA in humans and chimpanzees. Molecular Biology and Evolution 10 (3): 512-526. PMID 8336541.

Librado, P. and Rozas, J. (2009). DnaSP v5: A software for comprehensive analysis of DNA polymorphism data. Bioinformatics 25: 1451-1452.

Darriba, D., Taboada, G.L., Doallo, R., Posada, D. (2012). jModelTest 2: more models, new heuristics.

Drummond, A.J., Suchard, M.A., Xie, D. and Rambaut, A. (2012). Bayesian phylogenetics with BEAUti and the BEAST 1.7 Molecular Biology And Evolution 29: 1969-1973.

Rambaut, A., Suchard, M.A., Xie, D. and Drummond, A.J. (2014) Tracer v1.6, Available from http://beast.bio.ed.ac.uk/Tracer.

Wayne, R.K., Geffen, E., Girman, D.J., Koepfli, K.P., Lau, L.M., et al. (1997) Molecular systematics of the Canidae. Systematic Biology 46: 622-653. 
Vilà, C., Amorim, I.R., Leonard, J.A. , Posada, D., Castroviejo, J., Petrucci-Fonseca, F., Crandall, K.A., Ellegren, H., Wayne, R.K. (1999) Mitochondrial DNA phylogeography and population history of the grey wolf Canis lupus. Molecular Ecology 8, 2089-2103.

Lindblad-Toh, K., Wade, C., Mikkelsen, T.S., Karlsson, E.K., Jaffe, D.B., Kamal, M., Clamp, M., Chang, J.L., Kulbokas, E.J., Zody, M.C., et al. (2005). Genome sequence, comparative analysis and haplotype structure of the domestic dog. Nature 438: 803-819.

Vasco, J. (2012). Evolution and Biogeography of Canids (Canis and Vulpes) in North-West Africa. Facultade de Ciências Universidade Do Porto. Centro de Investigaçao em Biodiversidade e Recursos Geneticos.

Rueness, E. K., Trosvik, P., Atickem, A., Sillero-Zubiri, C., and Trucchi, E. (2015). The African wolf is a missing link in the wolf-like canid phylogeny. bioRxiv doi: http://dx.doi.org/10.1101/017996.

Bradley, R.D., Bake, R.J. (2001) A test of the genetic species concept: cytochrome-b sequences and mammals. Journal of Mammalogy 82: 960-973.

Hemprich, F. W. and C. G. Ehrenberg (1828-1833). Symbolae physicae seu icones et descriptiones mammalium. Decas 1.1828 (plates published y Sherborn., part of text not until 1833). Decas 2. although dated 1830 fide C. D.

Oken, L. (1815-1816). Lehrbuch der Naturgeschichte. Vol 3: Zoologie. Jena: August Schmidt und Comp.

Schwarz, V.E. (1926). ber typenexemplare von schakalen. Senckenbergiana 8: 39-47.

Ellerman, J.R. and Morrison-Scott, T.C.S. (1951). Checklist of Palaeartic and Indian Mammals 1758-1946.

Corbet, G.B. (1978). The Mammals of the Palaearctic Region: a Taxonomic Review. London and Ithaca: Cornell University Press.

Osborn Dale, J. and Helmy, I. (1980). The Contemporary Land Mammals from Egypt (Including Sinai). Fieldiana Zoology, New Series 5. 579 p. 164 illustr. 58 tables. 
Hocnaki, J. H., Inman, K.E. and Koeppl, J.W. (Eds). (1982). Mammal Species of the World: a taxonomy and geographic reference. Lawrence, Kansas, Allen Press, Inc. and The Association of Systematics Collections.

Wozencraft, W.C. (2005). Carnivora: Caniformia: Canidae. In (Wilson D.E. and D.M. Reeder Eds.) Mammal Species of the World: A Taxonomic. and Geographic Reference. Baltimore: The Johns Hopkins University Press.

Huxley, T.H. (1880). On the cranial and dental characters of the Canidae. Proceedings of the Zoological Society of London 125: 238-288

Ferguson, W.W. (1981). The systematic of Canis aureus lupaster (Carnivora: Canidae) and the occurrence of Canis lupus in North Africa, Egypt and Sinai. Mammalia 45 (4): 459-465.

Geraads, D. (2011). A revision of the fossil Canidae (Mammalia) of north-western Africa. Palaeontology, 54 (2), 429-446.

Anderson, J. (1902). Zoology of Egypt: Mammalia. London: Hugh. Rees.

Hilzheimer, M. (1908) Beitrag zur Kenntnis der Nordafrikanischen Schakale nebst Bemerkungen uber deren Verhaltnis zu den Haushunden insbesondere Nordafrikanischen und Altagyptischen Hunderassen. Zoologica. H53.

Flower, S. S. (1932): Notes on the recent mammals of Egypt, with a list of the species recorded from that Kingdom. Proc. Zool. Soc., London, 1932:368-450.

Kurten, B. (1965). The Carnivora of the Palestine Caves. - Acta Zool. Fennica, 107, 1-74.

Spassov N, (1989) The position of jackals in the Canis Genus and life-history of the golden jackal (Canis aureus L.) in Bulgaria and on the Balkans. Historia naturalis bulgarica. 44-56. Sofiia :Bulgarska akademiia na naukite, c1989- http://www.biodiversit.

Kowalski, K. and Rzebik-Kowalska, B. (1991). Mammals of Algeria. Krakow: Polish Academy of Sciences.

Heim de Balsac, H. (1936). Biogéographie des mammifères et des oiseaux de l'Afrique du Nord. Bulletin Biologique de la France et de la Belgique. A.1622:1-446. 
Geoffroy Saint-Hilaire, E., and Cuvier, F. (1824). Histoire Naturelle des Mammife` res, Tome Deuxie`me (Chez A. Belin).

Cabrera, Angel. (1932). Los mamíferos de Marruecos. Junta para Ampliación de Estudios e Investigaciones Científicas.; Museo Nacional de Ciencias Naturales. Madrid. España. 